\title{
Crosswind Kite Control - A Benchmark Problem for Advanced Control and Dynamic Optimization
}

\author{
Sean Costello, Grégory Françoisł and Dominique Bonvin
}

Version 3.0: 17.07.2016

\begin{abstract}
This article presents a kite control and optimization problem intended as a benchmark problem for advanced control and optimization. We provide an entry point to this exciting renewable energy system for researchers in control and optimization methods looking for a realistic test bench, and/or a useful application case for their theory. The benchmark problem in this paper can be studied in simulation, and a complete Simulink model is provided to facilitate this. The simulated scenario, which reproduces many of the challenges presented by a real system, is based on experimental studies from the literature, industrial data and the first author's own experience in experimental kite control. In particular, an experimentally validated wind turbulence model is included, which subjects the kite to realistic disturbances. The benchmark problem is that of controlling a kite such that the average line tension is maximized. Two different models are provided: A more comprehensive one is used to simulate the 'plant', while a simpler 'model' is used to design and implement control and optimization strategies. This way, uncertainty is present in the form of plant-model mismatch. The outputs of the plant are corrupted by measurement noise. The maximum achievable average line tension for the plant is calculated, which should facilitate the performance comparison of different algorithms. A simple control strategy is implemented on the plant and found to be quite sub-optimal, even if the free parameters of the algorithm are well tuned. An open question is whether or not more advanced control algorithms could do better.
\end{abstract}

\footnotetext{
${ }^{*}$ Dr. Costello (sean.c.costello@gmail.com) was with the Laboratoire d'Automatique, EPFL, Switzerland. He is currently with Leica Geosystems, St-Gallen, Switzerland.

${ }^{\dagger}$ Dr. François (gregory.francois@ed.ac.uk) was with the Laboratoire d'Automatique, EPFL, Switzerland. He is currently with the Institute of Material and Processes, School of Engineering, The University of Edinburgh, UK.

¥Prof. Bonvin (dominique.bonvin@epfl.ch) is with the Laboratoire d'Automatique, EPFL, Switzerland.
} 


\section{Contents}

1 Introduction $\quad 2$

2 State of the Art: Kite Control and Optimization 3

2.1 Control during crosswind flight . . . . . . . . . . . . . . 4

2.2 Trajectory optimization during crosswind flight . . . . . . . . 5

3 Modeling $\quad \mathbf{5}$

3.1 Dynamic equations for the plant . . . . . . . . . . . 6

3.2 How to simulate the plant . . . . . . . . . . . . . 10

4 Control Problem 11

4.1 Optimal control problem . . . . . . . . . . . . . . . . 11

4.2 Available measurements . . . . . . . . . . . . . . . . . 12

4.3 Control model . . . . . . . . . . . . . . . . . . 12

4.4 Validation of the control model . . . . . . . . . . . . 13

5 Performance of a Standard Control Algorithm 16

$\begin{array}{lll}6 & \text { Conclusion } & 17\end{array}$

7 Nomenclature $\quad 19$

A Further Modeling Details for the Simulated Reality 20

A.1 Lift and drag coefficients . . . . . . . . . . . . . . . . . . 20

A.2 Wind model . . . . . . . . . . . . . . . . . . . 21

\section{Introduction}

The contribution of this article is to present a challenging benchmark problem for control and optimization. The system used for the benchmark is the control of a power kite. This is both an exciting renewable energy system, and a difficultto-control nonlinear system. What is more, it naturally requires optimization to maximize the energy output. In this article, we aim to provide an entry point to this system for researchers in control and optimization methods looking for a realistic test bench, and/or a useful application case for their theory.

The control and optimization challenges inherent in kite power have received much attention from the research community in recent years (see $[22,1]$ for a comprehensive review of developments). In its essence, kite power aims to harness the aerodynamic forces a wing generates when flying almost perpendicular to the wind. As the kite flies very fast (many times the wind speed) and it is tethered to the ground, it must frequently change direction to avoid crashing. Hence, the kite will in general follow a periodic path. The control engineer is confronted with two interesting interrelated problems, namely, path planning (optimization) and path following (control). Controlling the kite autonomously such that it flies a suitable path is a challenging control problem. From a control point of view, difficulties stem from the lack of precise models, nonlinear behavior, fast unstable dynamics, measurement noise and large perturbations due to the wind. Path planning is important because the kite path directly 
determines the aerodynamic forces the kite experiences, and hence the power generated. Control design and optimization are usually performed using simplified dynamic models that are more computationally tractable and intuitive. However, it is well known that accurately modeling a kite behavior requires extremely detailed models $[24,8]$, and these simplified models are unavoidably inaccurate. Even early in the field of kite control, this motivated the design of robust control strategies, see for example [27, 30]. The control and optimization practitioner must thus face the problem of plant-model mismatch, that is, the model used to design the control strategy does not quite match the reality.

The aim of this paper is to present a challenging benchmark problem for control and optimization of a fast dynamic system to the control and optimization community. The benchmark scenario includes the difficulties of dealing with stochastic disturbances and plant-model mismatch. This paper uses a plausible simulated reality referred to as the 'plant'. The plant-model mismatch is plausible, yet artificial (since the plant is itself a model). This approach has long been used to benchmark measurement-based real-time optimization techniques in simulation, see for example [10]. The plant presented here does not aspire to perfectly mimic reality, and hence it can be represented using reasonably simple equations. This makes the benchmark easily reproducible. Nonetheless, every effort was made to ensure the benchmark includes many of the same trade-offs and challenges as the real problem. To this end, the authors used practical insight gained during several years of kite control experiments. The equations of both the simulated reality and the control model are either based on well-established first-principles modeling, or have been validated experimentally. Realistic parameter values were chosen for the plant, based on data for real systems. One indication of the validity of these models is that they both predict a qualitatively similar optimal path for the kite: a figure-of-eight, which has been confirmed by much more complex models.

The relative simplicity of the equations for the plant and the model is intended to allow researchers without expertise in kite power to easily apply their algorithms in a rigidly defined, reasonably realistic setting, and be confronted by many of the challenges inherent in a real system. For such a reader, this paper is intended to be a self-contained reference, for which a Simulink implementation of the simulated reality is provided.

\section{State of the Art: Kite Control and Optimiza- tion}

The control of kites is one of the most significant technical barriers that need to be actively tackled in order for kite power to become commercially viable. In order to extract maximum power from the wind, a power kite is flown almost perpendicular to the wind, similarly to the blades of a wind turbine, reaching speeds many times that of the wind itself (easily in excess of $150 \mathrm{~km} / \mathrm{h}$ ). This is known as crosswind flight, and is the flight mode considered in this paper. During crosswind flight, to avoid crashing in a matter of seconds, an "autopilot" must keep the kite flying in a wide variety of wind conditions, thus providing stability. In addition to keeping the kite from crashing, the autopilot should ensure the kite follows a path that is efficient for power production. 
Before reviewing the literature in more detail in the following sections, we provide a brief summary of the state of the art. Due to the difficulty of the control task (nonlinearity, model uncertainty, large disturbances, noisy measurements), advanced control approaches have long been envisaged, and some have been tested. However, in practice, simple semi-heuristic control strategies continue to dominate. We believe that this will change in the coming years, as there exists a significant potential for improvement through more advanced control strategies. This will hopefully lead to (i) improved and more flexible tracking capabilities, (ii) improved robustness, and (iii) the possibility to optimize efficiency in real time. A very promising control strategy that has yet to be fully exploited in this context is model predictive control (MPC). We also see much potential for geometric approaches that account for the kite being constrained to a spherical surface.

\subsection{Control during crosswind flight}

The principal objective of kite control during crosswind flight is to steer the kite such that it follows the prescribed figure-of-eight as robustly as possible. Although in some situations there are secondary manipulated variables such as tether winch tension, or the kite's pitch angle, these fall outside the scope of this article. Large deviations from the prescribed path can result in: a) crashing, which is obviously very undesirable, b) entering a very low-power area of the wind window and losing speed due to an unfavorable angle between the kite's lines and the wind, possibly causing a loss of controllability or a stall, c) entering a very high-power area of the wind window, which in strong winds can lead to material failure. The main compounding factors are variable wind speed (which leads to variable kite speed and steering behavior), noisy measurements, and delay in the control loop.

The field of kite control is young, yet varied. To date, there has been a focus on relatively straightforward, practical methods. This is helped by the fact that, during crosswind flight, it is often desirable that the kite follows a horizontally lying figure-of-eight pattern. Both [19] and [23] developed simple, robust, cascade controllers for power kites that produce a figure-of-eight pattern. A low-level proportional controller regulates the kite's direction of motion (steering), while a higher-level guidance controller alternately directs the kite towards one of two points, or zones. Variants of these algorithms have been extensively tested on a number of systems (see for example [41, 21]), and can perhaps be considered the standard approach for practical kite control.

Given the spherical surface upon which the kite flies, geometric approaches to control design are very attractive. For example, [5] observed that a simple control scheme should aspire to control the kite's direction of motion, referred to as the velocity angle. Lining the velocity angle to the concept of geodesic curvature elegantly simplifies the problem of tracking on a sphere. Building on the work by [5], [31] proposed a more advanced path-following controller using feedback linearization and an elegant nonlinear guidance law, and successfully tested it on a $20-\mathrm{kW}$ pumping-cycle prototype. Successful prototype implementations of geometrically motivated path-following controllers are also reported by [34] and [11]. 


\subsection{Trajectory optimization during crosswind flight}

The path taken by the kite is directly linked to the power extracted from the wind. As the kite will typically fly a figure-of-eight during crosswind flight, this leaves the shape, size and position of the figure of eight to be defined. The objective is usually to maximize the average useful power extracted from the wind. The optimization cost function will vary depending on the specific system and on whether the application is electricity generation or vehicle propulsion, but the instantaneous power is always proportional to the tether tension. Any optimization formulation must take into account operating constraints, such as height limits or steering-actuator bounds. However, the main difficulty stems from the kite's poorly modeled behavior and the wind variability. This means that the optimal path cannot be simply computed once off line, but should really be updated in real time to take into account wind variations (in particular of the vertical profile) and modeling inaccuracies.

While several control solutions for kites now exist, the path optimization problem during crosswind flight is still an open issue. Intelligent path planning is recognized to be extremely important, and experimental studies have confirmed that the path taken by the kite significantly affects the power it can generate [39, 11]. The optimal control problem is quite well understood, and has been studied from a theoretical perspective by a number of authors $[17,28,29,38,4,16]$. However, despite promising recent work [26, 18], there remains a gap between theory and application. Very recently, experimental approaches have begun to be investigated to tune the path the kite follows in real-time: [39, 40, 11]. These form an optimization layer that sits above the control layer in the architecture. As an approach that solves the control and optimization problem simultaneously, state-of-the-art nonlinear model predictive control (NMPC) would appear the perfect solution $[17,30,9]$. Although there have been exciting recent advances [36], this has unfortunately yet to become a practical reality, probably mainly due to the inaccuracy of existing kite models, as NMPC relies on the quality of the model at hand. It remains to be demonstrated whether a MPC controller based on a simple control model can handle the plant-model mismatch that is bound to occur in practice.

\section{Modeling}

The plant consists of a kite (i.e. a wing) attached to a fixed point by a fixedlength tether. A wealth of information on modeling kite dynamics has become available in the last decade $[17,28,15,7,20,24,25,8,6,18]$. Models can be constructed with anything from 3 to several hundred states. However, since the aerodynamics of a kite are (i) very difficult to model precisely, and (ii) very dependent on the kite design, even the most detailed models cannot claim to be perfect. In this paper, the equations for the simulated reality represent a fair compromise between detail and complexity. We build upon the well-established point-mass model, relying upon [17] and [23]. The co-ordinate system is chosen to be compatible with the control model discussed in Section 4.3. We include embellishments of the basic point-mass dynamics, account for changes in the angle-of-attack, wind shear and wind gusting, and consider the dynamics of the steering actuator. 


\subsection{Dynamic equations for the plant}

The fixed, inertial, right-hand co-ordinate system $G$ is depicted in Figure 1. The $\mathrm{x}$ and $\mathrm{y}$ axes are horizontal, while the z-axis points skywards. The kite position

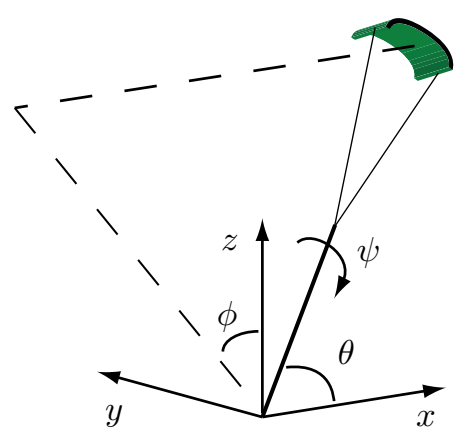

Figure 1: Inertial co-ordinate system $G$.

$\mathbf{p}$ in cartesian $\{x, y, z\}$ co-ordinates (using the $\mathrm{x}$-axis as the zenith) is:

$$
\mathbf{p}=\left[\begin{array}{c}
p_{x} \\
p_{y} \\
p_{z}
\end{array}\right]=r\left[\begin{array}{c}
\cos \theta \\
\sin \theta \sin \phi \\
\sin \theta \cos \phi
\end{array}\right]
$$

where the spherical coordinates $(\theta, \phi, r)$ are the polar angle, azimuthal angle and radial distance. Differentiating this yields the kite velocity in terms of the spherical co-ordinates and their derivatives:

$$
\dot{\mathbf{p}}=\left[\begin{array}{ccc}
-r \sin \theta & 0 & \cos \theta \\
r \cos \theta \sin \phi & r \cos \phi \sin \theta & \sin \phi \sin \theta \\
r \cos \phi \cos \theta & -r \sin \phi \sin \theta & \cos \phi \sin \theta
\end{array}\right]\left[\begin{array}{c}
\dot{\theta} \\
\dot{\phi} \\
\dot{r}
\end{array}\right] .
$$

We define a local, non-inertial, right-hand co-ordinate system $L_{1}$ from the basis vectors $\left\{\hat{\mathbf{e}}_{\theta}, \hat{\mathbf{e}}_{\phi}, \hat{\mathbf{e}}_{\text {down }}\right\}$ given by:

$$
\hat{\mathbf{e}}_{\theta}=\frac{\frac{\partial \mathbf{p}}{\partial \theta}}{\left|\frac{\partial \mathbf{p}}{\partial \theta}\right|}, \quad \hat{\mathbf{e}}_{\phi}=\frac{\frac{\partial \mathbf{p}}{\partial \phi}}{\left|\frac{\partial \mathbf{p}}{\partial \phi}\right|}, \quad \hat{\mathbf{e}}_{\mathrm{down}}=-\frac{\frac{\partial \mathbf{p}}{\partial r}}{\left|\frac{\partial \mathbf{p}}{\partial r}\right|} .
$$

Hence, the mapping from $L_{1}$ to $G$ is given by the rotation matrix:

$$
L_{1} \rightarrow G:\left[\begin{array}{lll}
\hat{\mathbf{e}}_{\theta} & \hat{\mathbf{e}}_{\phi} & \hat{\mathbf{e}}_{\mathrm{down}}
\end{array}\right]
$$

and the inverse of this matrix is the mapping from $G$ to $L_{1}$. The subscript $L_{1}$ will precede a vector (matrix) to indicate that the vector (matrix) is expressed in $L_{1}$ (maps $L_{1}$ to $L_{1}$ ). The kite velocity is

$$
L_{1} \dot{\mathbf{p}}=\left[\begin{array}{c}
\dot{\theta} r \\
\dot{\phi} r \sin \theta \\
-\dot{r}
\end{array}\right]
$$


and differentiating once more with respect to time, Newton's law reads:

$$
\frac{L_{1} \mathbf{F}}{m}={ }_{L_{1}} \ddot{\mathbf{p}}=\left[\begin{array}{c}
2 \dot{r} \dot{\theta}+\ddot{\theta} r-\frac{\dot{\phi}^{2} r \sin (2 \theta)}{2} \\
2 \dot{\phi} \dot{r} \sin \theta+\ddot{\phi} r \sin \theta+2 \dot{\phi} \dot{\theta} r \cos \theta \\
\dot{\theta}^{2} r-\ddot{r}+\dot{\phi}^{2} r \sin ^{2} \theta
\end{array}\right] .
$$

Assuming the tether is straight (which is an often-made and usually reasonable approximation for crosswind flight), $\dot{r}=0$ and $\ddot{r}=0$, and we can solve for the acceleration of the kite in spherical co-ordinates and for the overall force in the radial direction:

$$
\begin{aligned}
& \ddot{\theta}=\frac{m r \sin (2 \theta) \dot{\phi}^{2}+2 \mathbf{F} \cdot \hat{\mathbf{e}}_{\theta}}{2 m r}, \\
& \ddot{\phi}=\frac{\mathbf{F} \cdot \hat{\mathbf{e}}_{\phi}-2 \dot{\phi} \dot{\theta} m r \cos \theta}{m r \sin \theta}, \\
& \mathbf{F} \cdot \hat{\mathbf{e}}_{\text {down }}=m r\left(\dot{\phi}^{2} \sin ^{2} \theta+\dot{\theta}^{2}\right) .
\end{aligned}
$$

The next step is to obtain expressions for the forces acting on the kite. The gravitational force acting on the kite is:

$$
\mathbf{F}_{\mathrm{g}}=\left[\begin{array}{c}
0 \\
0 \\
-m g
\end{array}\right] \text {. }
$$

The aerodynamic force depends on the apparent wind and the kite orientation. The apparent wind is defined as

$$
\mathbf{w}_{\mathrm{a}}=\mathbf{w}-\dot{\mathbf{p}},
$$

where $\mathbf{w}$ is the wind vector. We define the projected apparent-wind vector as

$$
L_{1} \mathbf{w}_{\text {ap }}=\left[\begin{array}{ccc}
1 & 0 & 0 \\
0 & 1 & 0 \\
0 & 0 & 0
\end{array}\right] L_{1} \mathbf{w}_{\mathrm{a}} .
$$

This is the projection of the apparent wind onto the plane tangent to the sphere at the kite position. We now define $L_{2}$, a further non-inertial, right-hand, local co-ordinate frame with the basis vectors $\left\{\hat{\mathbf{e}}_{\mathrm{f}}, \hat{\mathbf{e}}_{0}, \hat{\mathbf{e}}_{\text {down }}\right\}$, where

$$
\hat{\mathbf{e}}_{\mathrm{f}}=-\frac{\mathbf{w}_{\mathrm{ap}}}{\left|\mathbf{w}_{\mathrm{ap}}\right|}, \quad \hat{\mathbf{e}}_{0}=\hat{\mathbf{e}}_{\mathrm{down}} \times \hat{\mathbf{e}}_{\mathrm{f}} .
$$

Using $L_{2}$ simplifies the analysis of the kite orientation because the apparent wind vector is perpendicular to $\hat{\mathbf{e}}_{0}$. Again, the subscript $L_{2}$ will precede a vector (matrix) to indicate that the vector (matrix) is expressed in $L_{2}$ (maps $L_{2}$ to $L_{2}$ ). The orientation of the kite can be represented using two vectors: as shown in Figure 2, $\hat{\mathbf{e}}_{\text {pitch }}$ points along the right wing, perpendicular to $\hat{\mathbf{e}}_{\text {roll }}$ that points forwards. To find the orientation of these vectors, we begin by aligning the kite with the axes of the $L_{2}$ frame (this initial frame is denoted with a 0 ), and then apply two successive rotations (where the intermediate frame is denoted 1):

$$
L_{2} \hat{\mathbf{e}}_{\mathrm{pitch}}^{0}=\left[\begin{array}{l}
0 \\
1 \\
0
\end{array}\right], \quad L_{2} \hat{\mathbf{e}}_{\mathrm{roll}}^{0}=\left[\begin{array}{l}
1 \\
0 \\
0
\end{array}\right] .
$$




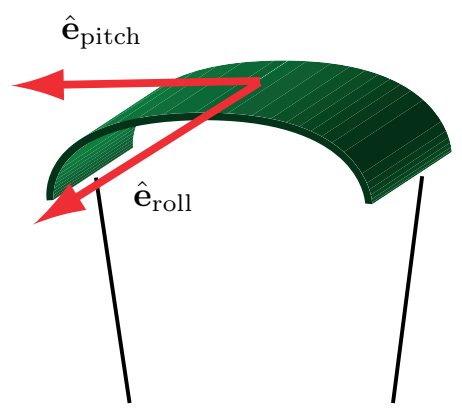

Figure 2: Kite orientation vectors.

In the absence of a steering deflection, this is the kite orientation. However, a steering deflection rotates the kite around $\hat{\mathbf{e}}_{\text {roll }}$ in the following manner:

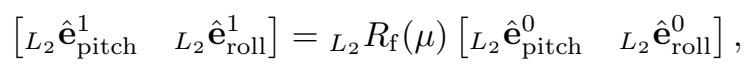

where $L_{2} \mathbf{R}_{\mathrm{f}}(\mu)$ is a rotation (in $L_{2}$ ) around the $\hat{\mathbf{e}}_{\mathrm{f}}$-axis in the sense of a righthand corkscrew:

$$
{ }_{L_{2}} \mathbf{R}_{\mathrm{f}}(\mu)=\left[\begin{array}{ccc}
1 & 0 & 0 \\
0 & \cos \mu & -\sin \mu \\
0 & \sin \mu & \cos \mu
\end{array}\right],
$$

and the value of $\mu$ is given by

$$
\mu=\sin ^{-1}(-\bar{u} / d)
$$

where $\bar{u}$ is the steering deflection and $d$ is the wingspan. In reality, the steering deflection cannot be varied instantaneously, so the manipulated variable $u$ is the set point for the steering deflection. We assume a first-order relationship between the two (which is typical for a correctly tuned motor-actuated control loop adjusting the steering deflection):

$$
\dot{\bar{u}}=\frac{1}{\tau_{\mathrm{u}}}(u-\bar{u}) .
$$

We make the classic 'infinite-tail' assumption [17], which states that the kite will point into the apparent wind. More precisely, $\hat{\mathbf{e}}_{\text {pitch }}$ is always perpendicular to the apparent wind. The kite will satisfy this condition by rotating an angle $\eta$ around $\hat{\mathbf{e}}_{\text {down }}$ :

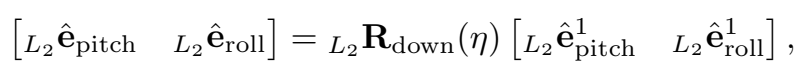

where $L_{2} R_{\text {down }}(\eta)$ is a counter-clockwise rotation around the $\hat{\mathbf{e}}_{\text {down-axis: }}$

$$
{ }_{L_{2}} \mathbf{R}_{\text {down }}(\eta)=\left[\begin{array}{ccc}
\cos \eta & -\sin \eta & 0 \\
\sin \eta & \cos \eta & 0 \\
0 & 0 & 1
\end{array}\right] .
$$

According to the 'infinite-tail' assumption, $\hat{\mathbf{e}}_{\text {pitch }}$ must satisfy

$$
\hat{\mathbf{e}}_{\mathrm{pitch}} \cdot \mathbf{w}_{a}=0 \text {. }
$$


Using the expression for $L_{2} \hat{\mathbf{e}}_{\text {pitch }}$ obtained from Equations (3.14), (3.15), (3.19), and noting that $\mathbf{w}_{\mathrm{a}} \cdot \hat{\mathbf{e}}_{0}=0$, we can solve for $\eta$ :

$$
\eta=\sin ^{-1}\left(\frac{\mathbf{w}_{\mathrm{a}} \cdot \hat{\mathbf{e}}_{\mathrm{down}} \tan \mu}{\mathbf{w}_{\mathrm{a}} \cdot \hat{\mathbf{e}}_{\mathrm{f}}}\right) .
$$

When the kite is moving slowly, $\mathbf{w}_{\mathrm{a}} \cdot \hat{\mathbf{e}}_{\mathrm{f}}$ is relatively small. In this situation, a large steering deflection, and hence a large value of $\tan \mu$, could result in Equation (3.22) returning an imaginary value of $\eta$. This means the kite cannot physically point into the apparent wind, which violates the assumption made by the plant model. In reality, as the kite is designed to fly aligned with the apparent wind, such a steering input will generally result in a loss of control. In the simulated reality, it is considered that applying such an input results directly in a crash.

The direction in which the kite is pointing can be represented using the heading angle $\psi$, defined as the angle from $\hat{\mathbf{e}}_{\theta}$ to $\hat{\mathbf{e}}_{\text {roll }}$, moving clockwise around

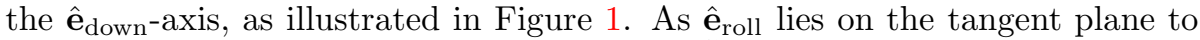
the sphere, a 'wrapped' version of this angle, $\tilde{\psi} \in[-\pi / 2, \pi / 2]$, can be found by inverting the relationship:

$$
\tan \tilde{\psi}=\frac{\left(-\hat{\mathbf{e}}_{\text {roll }} \cdot \hat{\mathbf{e}}_{\phi}\right)}{\left(\hat{\mathbf{e}}_{\mathrm{roll}} \cdot \hat{\mathbf{e}}_{\theta}\right)}
$$

taking care to choose $\tilde{\psi}$ in the correct quadrant (for example, using the matlab function 'atan2'). 'Unwrapping' this angle (for example using the matlab function 'unwrap') gives $\psi$ :

$$
\psi(t)=\int_{0}^{t_{1}^{-}} \dot{\tilde{\psi}} d t+\sum_{i=1}^{N-1}\left(\int_{t_{i}^{+}}^{t_{i+1}^{-}} \dot{\tilde{\psi}} d t\right)+\int_{t_{N}^{+}}^{t} \dot{\tilde{\psi}} d t
$$

where $\left\{t_{1}, \ldots t_{N}\right\} \in[0, t]$ are the time instants at which $\tilde{\psi}(t)$ is discontinuous (due to a jump of $2 \pi$ ).

Now that the kite orientation is known, we can calculate the aerodynamic force. This force is conditioned by the angle of attack, denoted $\alpha$, which is the angle between the $\hat{\mathbf{e}}_{\text {roll }}$ and $\mathbf{w}_{\mathrm{a}}$ :

$$
\mathbf{F}_{\text {aero }}=\left(\frac{1}{2} \rho A\left|\mathbf{w}_{\mathrm{a}}\right|^{2}\right)\left(C_{\mathrm{L}}(\alpha) \hat{\mathbf{w}}_{\mathrm{a}} \times \hat{\mathbf{e}}_{\text {pitch }}+C_{\mathrm{D}}(\alpha) \hat{\mathbf{w}}_{\mathrm{a}}\right),
$$

where $\rho$ is the air density and $\hat{\mathbf{w}}_{\mathrm{a}}$ is a unit vector pointing in the direction of the apparent wind and $C_{L}(\alpha)$ and $C_{\mathrm{D}}(\alpha)$ are the lift and drag coefficients, both of which are functions of the angle of attack ${ }^{1}$. The sum of the forces on the system is

$$
\mathbf{F}=\mathbf{F}_{\text {aero }}+\mathbf{F}_{\mathrm{g}}+T \hat{\mathbf{e}}_{\text {down }},
$$

where $T$ is the tether tension, given by

$$
T=\mathbf{F} \cdot \hat{\mathbf{e}}_{\text {down }}-\left(\mathbf{F}_{\text {aero }}+\mathbf{F}_{\mathrm{g}}\right) \cdot \hat{\mathbf{e}}_{\text {down }} .
$$

\footnotetext{
${ }^{1}$ The drag coefficient given here should be considered that of the overall system, including the tether. This model does not separately model tether drag, as we do not consider this necessary. For crosswind flight, which is the focus of this article, it is reasonable to lump the the total system drag into one drag coefficient, as in $[28,20]$.
} 
Table 1: Plant parameter values.

\begin{tabular}{|c|c|l|}
\hline Parameter & Value & Units \\
\hline$r$ & 250 & $\mathrm{~m}$ \\
$A$ & 25 & $\mathrm{~m}^{2}$ \\
$d$ & 10 & $\mathrm{~m}$ \\
$m$ & 300 & $\mathrm{~kg}$ \\
$g$ & 9.8 & $\mathrm{~m} \cdot \mathrm{s}^{-2}$ \\
$\rho$ & 1.2 & $\mathrm{~kg} \cdot \mathrm{m}^{-3}$ \\
$z_{\text {ref }}$ & 10 & $\mathrm{~m}$ \\
$w_{\text {ref }}$ & 8 & $\mathrm{~m} \cdot \mathrm{s}^{-1}$ \\
$a$ & .15 & - \\
$\chi$ & 15 & $\mathrm{degrees}$ \\
$T_{\mathrm{w}}$ & .5 & $\mathrm{~s}$ \\
$T_{\mathrm{S}}$ & .125 & $\mathrm{~s}$ \\
$k_{\sigma, \mathrm{w}}$ & 0.14 & - \\
$L_{\mathrm{w}}$ & 100 & $\mathrm{~m}^{2} \cdot \mathrm{m}^{-2}$ \\
$\sigma_{\mathrm{w}}^{2}$ & 2 & $\mathrm{~m}^{2} \cdot \mathrm{rad}^{2}$ \\
$\sigma_{\theta}^{2}$ & $1 \times 10^{-4}$ & $\mathrm{rad}^{2}$ \\
$\sigma_{\phi}^{2}$ & $1 \times 10^{-4}$ & $\mathrm{rad}^{2}$ \\
$\sigma_{\psi}^{2}$ & .02 & $\mathrm{rad}^{2}$ \\
$C_{\mathrm{L}, 0}$ & .57 & - \\
$C_{\mathrm{L}, 1}$ & 1.547 & - \\
$C_{\mathrm{D}, 0}$ & .11 & - \\
$C_{\mathrm{D}, 2}$ & 1.168 & - \\
\hline
\end{tabular}

\subsection{How to simulate the plant}

The parameters for the simulated reality are given in Table 1. Although no source discloses the full specifications of a large-scale kite system (mainly because they are mostly in the development phase), the parameters were selected to correspond reasonably well to a number of existing or developing systems $[35,2,19,34]$. The following steps are performed to simulate the plant:

1. The initial conditions $\{\theta(0), \dot{\theta}(0), \phi(0), \dot{\phi}(0), \bar{u}(0)\}=\left\{\theta_{0}, \omega_{0}^{\theta}, \phi_{0}, \omega_{0}^{\phi}, \bar{u}_{0}\right\}$ specified in Table 2 correspond to the instant right after the kite is launched.

2. Compute the apparent wind vector based on the current position and velocity. Calculate the basis vectors for the two local co-ordinate frames, and the rotation matrices that let you go from them to $G$.

3. Calculate the angle $\eta$ using equation (3.22). Obtain the kite orientation by applying the rotations in Equations (3.16) and (3.20) to $\hat{\mathbf{e}}_{\text {pitch }}^{0}$ and $\hat{\mathbf{e}}_{\text {roll }}^{0}$.

4. Compute the aerodynamic and gravitational forces, which are the only forces acting in directions $\hat{\mathbf{e}}_{\theta}$ and $\hat{\mathbf{e}}_{\phi}$.

5. By inserting the values of the aerodynamic and gravitational forces into Equations (3.7)-(3.9), obtain the kite acceleration, and the radial force 
necessary to keep it on the sphere $\mathbf{F} \cdot \hat{\mathbf{e}}_{\text {down }}$. The line tension can be obtained using Equation (3.27). Return to step 2.

\section{Control Problem}

\subsection{Optimal control problem}

In continuous operation, the manipulated variable $u(t)$ (the set point for the steering deflection) should be used to maximize the average line tension, while respecting an altitude constraint. This problem is very similar to other kitepower optimization problems, such as maximizing the power generated while slowly reeling out the tether (in fact, in many studies it is assumed the reeling out speed is constant $[39,32,9])$, or maximizing the average force in a fixed direction. For a given time horizon, $t_{\mathrm{f}}$, the problem can be formulated as:

$$
\begin{array}{ll}
\underset{u(t)}{\operatorname{maximize}} & \bar{T}\left(t_{\mathrm{f}}\right):=\frac{1}{t_{\mathrm{f}}} \int_{0}^{t_{\mathrm{f}}} T(t) d t, \\
\text { subject to } & |u(t)| \leq u_{\max }, \\
& r \sin (\theta(t)) \cos (\phi(t)) \geq z_{\min }, \\
& |\psi(t)| \leq 2 \pi .
\end{array}
$$

The upper bound on the steering deflection, $u_{\max }$, ensures the kite roll angle does not exceed $37^{\circ}$. The last constraint is a winding constraint for the tether to ensure it is not twisted. The numerical values of these bounds are given in Table 3. Although not explicitly included in the optimization problem, the control scheme should also aspire to keep the input $u(t)$ as smooth as possible to reduce component wear.

The solution to the above optimal control problem will vary depending on the wind realization, the initial state of the plant, and the value of $t_{f}$. The initial conditions for the benchmark scenario are specified in Table 2. To ensure a fair comparison, the thrust $\bar{T}\left(t_{\mathrm{f}}\right)$ obtained using a particular controller should be averaged over a timespan of continuous operation that is much greater than the update rates of the stochastic wind disturbances. The time period used to evaluate a control algorithm should respect $t_{\mathrm{f}}>200 \mathrm{~s}$ to ensure the RMS wind speed is approximately constant for different realizations of the stochastic wind signal.

Table 2: Initial conditions for the benchmark scenario.

\begin{tabular}{|c|c|l|}
\hline Variable & Value & Units \\
\hline$\theta_{0}$ & 0.11 & $\mathrm{rad}$ \\
$\omega_{0}^{\theta}$ & 0.15 & $\mathrm{rad} \cdot \mathrm{s}^{-1}$ \\
$\phi_{0}$ & 0 & $\mathrm{rad}$ \\
$\omega_{0}^{\phi}$ & 0 & $\mathrm{rad} \cdot \mathrm{s}^{-1}$ \\
$\bar{u}_{0}$ & 0 & $\mathrm{~m}$ \\
\hline
\end{tabular}


Table 3: Operational bounds

\begin{tabular}{|c|c|l|}
\hline Parameter & Value & Units \\
\hline$z_{\min }$ & 25 & $\mathrm{~m}$ \\
$u_{\max }$ & 7.5 & $\mathrm{~m}$ \\
\hline
\end{tabular}

\subsection{Available measurements}

It is assumed that the following sampled, noisy measurement vector is available:

$$
\mathbf{y}_{\mathrm{N}}[k]=\left[\begin{array}{c}
\theta\left(k T_{\mathrm{s}}\right)+\theta_{\mathrm{N}}[k] \\
\phi\left(k T_{\mathrm{s}}\right)+\phi_{\mathrm{N}}[k] \\
\psi\left(k T_{\mathrm{s}}\right)+\psi_{\mathrm{N}}[k] \\
\int_{0}^{k T_{\mathrm{s}}} T(t) d t
\end{array}\right],
$$

where $T_{\mathrm{S}}$ is the sampling period, and $\theta_{\mathrm{N}}[k], \phi_{\mathrm{N}}[k]$ and $\psi_{\mathrm{N}}[k]$ are (for each $k$ ) drawn from a zero-mean Gaussian distribution with variances $\sigma_{\theta}^{2}, \sigma_{\phi}^{2}$ and $\sigma_{\psi}^{2}$, respectively. The variance values are given in Table 1 . The sampling period of $125 \mathrm{~ms}$ is suggested (which is reasonable for such a large system, where the kites position evolves relatively slowly compared to the tether length. This puts the problem well within reach of computationally intensive control algorithms such as NMPC. However, if necessary, it is certainly realistic to assume a sampling period as low as $10 \mathrm{~ms}$ is available, and this parameter can be easily modified in the Simulink model.

\subsection{Control model}

In the benchmark scenario, the underlying equations of the simulated reality are unknown, and only a control model is available. This is the simple and elegant 3 -state model developed by [19]. This tendency model has been successfully used to design control algorithms for very large kites. An embellishment proposed by [12] accounts for the reduction of line tension caused by steering deflections, a modification that is necessary to obtain meaningful solutions to the pathplanning problem.

The dynamic equations for the control model are simple analytic expressions, so they are simply stated here (the interested reader is invited to see [19] and [12] for more details):

$$
\begin{aligned}
& \dot{\theta}=\frac{w_{\mathrm{ap}}}{r}\left(\cos \psi-\frac{\tan \theta}{E}\right), \\
& \dot{\phi}=-\frac{w_{\mathrm{ap}}}{r \sin \theta} \sin \psi, \\
& \dot{\psi}=w_{\mathrm{ap}} g_{\mathrm{s}} u+\dot{\phi} \cos \theta,
\end{aligned}
$$

where the lift-to-drag ratio $E$ and the magnitude of the apparent wind projected onto the quarter sphere, $w_{\text {ap }}$, are given by

$$
\begin{aligned}
& w_{\text {ap }}=w_{0} E \cos \theta, \\
& E=E_{0}-c u^{2},
\end{aligned}
$$


where $w_{0}$ is the fixed wind-speed and $E_{0}$ is the effective lift-to-drag ratio for the control model. The line tension is given by

$$
T=\left(\frac{1}{2} \rho A w_{0}^{2}\right) \cos ^{2} \theta(E+1) \sqrt{E^{2}+1} .
$$

The parameters of the control model are given in Table 4. These parameters are estimated from the plant behavior, as they would be in reality, using the kite-specific parameter estimation steps given in [19].

Table 4: Model parameter values.

\begin{tabular}{|c|c|l|}
\hline Parameter & Nominal value & Units \\
\hline$r$ & 250 & $\mathrm{~m}$ \\
$A$ & 25 & $\mathrm{~m}^{2}$ \\
$E_{0}$ & 6 & - \\
$w_{0}$ & 11 & $\mathrm{~m} \cdot \mathrm{s}^{-1}$ \\
$g_{\mathrm{s}}$ & $5 \times 10^{-3}$ & $\mathrm{rad} \cdot \mathrm{m}^{-2}$ \\
$c$ & .06 & $\mathrm{~m}^{-2}$ \\
$\rho$ & 1.2 & $\mathrm{~kg} \cdot \mathrm{m}^{-3}$ \\
\hline
\end{tabular}

The control model is significantly different from the plant. Firstly, the control model assumes the wind is aligned with the $x$-axis (see Figure 1), while in the simulated reality, the wind experienced by the kite is offset from this axis by the angle $\chi$. This can easily occur in practice as the wind experienced by the kite is not measured directly, and may differ substantially from the wind vector measured on the ground. Secondly, the control model is a kinematic model; it does not take into account the inertial effects due to the kite mass. Thirdly, the control model assumes a constant wind velocity, irrespective of the kite altitude. In the simulated reality, there is both wind shear (variation with altitude) and stochastic turbulence.

\subsection{Validation of the control model}

How 'accurate' is the control model? This depends upon which aspect of the plant behavior the control model is used to approximate. Firstly, we study how well it can predict optimal operating conditions for the plant. For this, we consider the case where $t_{\mathrm{f}} \rightarrow \infty$ in (4.1), and assume that the optimal solution is periodic. We also use a deterministic wind-speed profile for the plant, the RMS profile, given by Equation (A.4) with $w_{\mathrm{N}}=0$. As the force a kite can generate flying in a particular direction is proportional to the square of the wind speed, solving the optimal control problem with the RMS wind profile should give an upper bound for the average thrust in continuous flight over a relatively long time period (at least $100 \mathrm{~s}$ ). The optimal (periodic) paths for both the plant and the control model are shown in Figure 3. As this analysis disregards noise and disturbances, the difference between the optimal paths is due to the significant plant-model mismatch. The optimal paths can be interpreted as follows:

- Both optimal paths have the classic 'figure-of-8' shape. For given wind conditions, there is an optimal position where the kite will tend to experi- 
ence the strongest aerodynamic force. The 'figure-of-eight' allows the kite to stay close to this position, while avoiding excessive steering deflections.

- The control model assumes a constant wind speed at all altitudes, and so the kite tries to fly as close to the downwind position $(z=y=0)$ as possible. Hence, the optimal path for the control model is limited by the minimum-altitude constraint.

- The plant optimal solution takes advantage of the wind gradient. The altitude of the 'figure-of-eight' is a compromise between accessing stronger winds at higher altitude and flying perpendicular to the wind direction.

- In addition, the control model assumes an incorrect wind direction, which explains the lateral offset between the two optimal paths.

The optimal average thrust for the plant is $\lim _{t_{\mathrm{f}} \rightarrow \infty} \bar{T}\left(t_{\mathrm{f}}\right)_{\mathrm{p}}^{*}=39.61 \mathrm{kN}$, and that for the control model is $\lim _{t_{\mathrm{f}} \rightarrow \infty} \bar{T}\left(t_{\mathrm{f}}\right)_{\mathrm{m}}^{*}=43.78 \mathrm{kN}$.

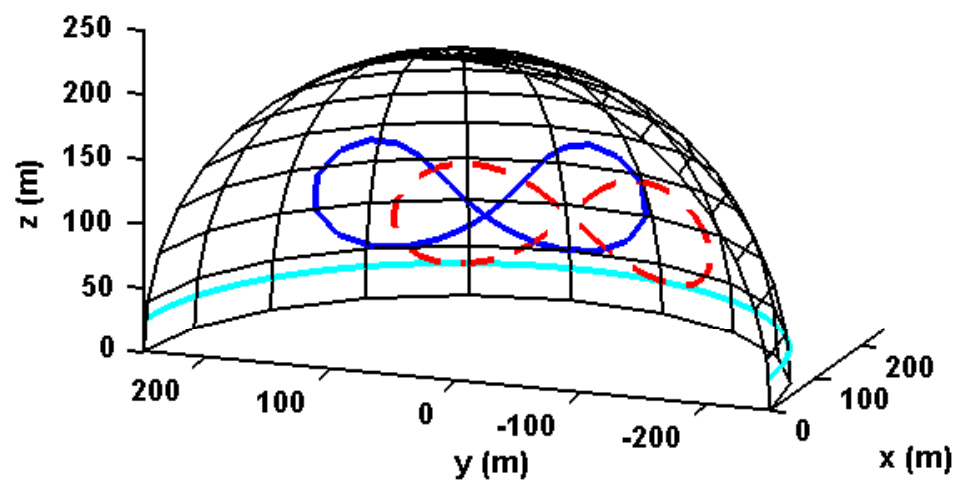

Figure 3: Optimal path for the plant (solid line, blue) with the root-meansquare wind-speed profile, and for the control model (dash line, red). The height constraint is shown in light blue.

It is also interesting to study how the states predicted by the control model and those of the plant diverge, starting from a common point. For this, we take a state trajectory for the plant, initialize the control model at different points along the trajectory, and integrate the control model equations forward in time. The results for the kite position are shown in Figure 4, and for the kite orientation in Figure 5. This illustrates why the control model should only be treated as a 'tendency' model: it predicts the general evolution of the system, but not accurately. Applying inputs computed using the control model in an open-loop fashion would clearly fail, hence the need for closed-loop control. The line tension estimated by the control model based on the plant states using Equation (4.11) is shown in Figure 6. At first sight the correlation between the control model and the plant seems very poor, however the input $u$ was very 
irregular in this case. The control model can be expected to produce better estimates of $T$ for smoother inputs. What is more, the fact that the optimal average tension calculated by the control model is very close to that of the plant restores some confidence in the control model capacity to predict the line tension.

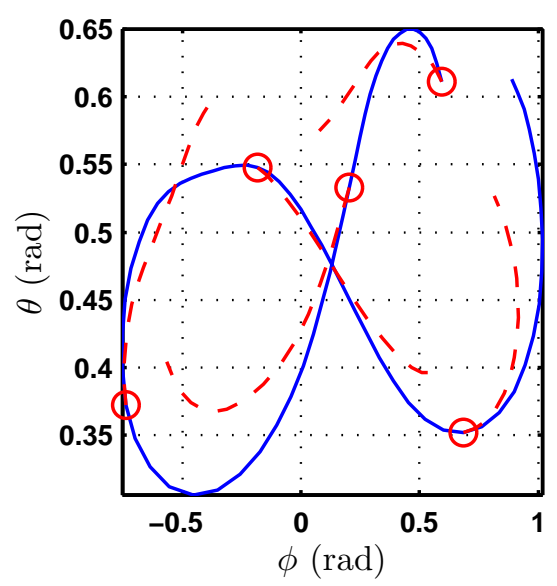

Figure 4: Plant trajectory (blue). The control model is provided with the plant states at each red circle, and integrated to 'predict' the plant evolution over the next $2 \mathrm{~s}$ (dashed red).

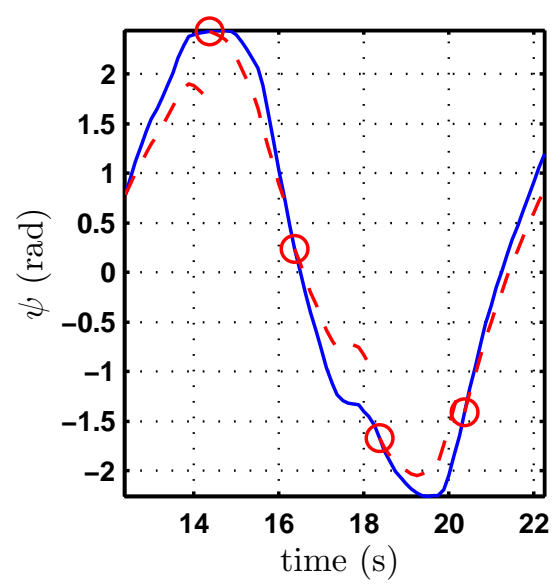

Figure 5: Plant heading angle (blue). The control model is provided with the plant states at each red circle, and integrated to 'predict' the plant evolution over the next $2 \mathrm{~s}$ (dashed red). 


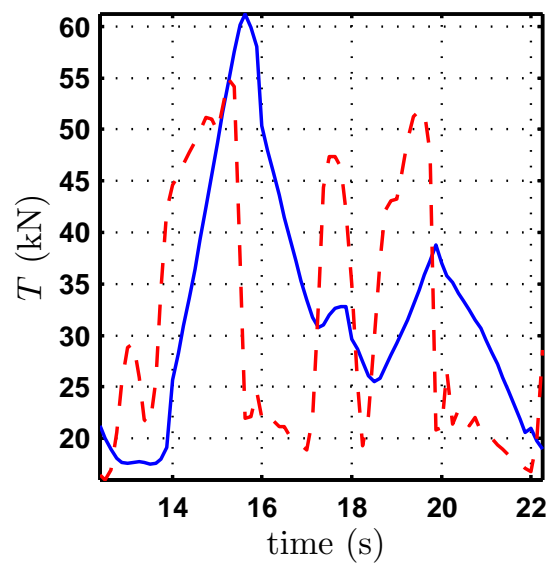

Figure 6: Plant tension (blue) and, in dashed red, the tension estimated with the control model from the plant states.

\section{Performance of a Standard Control Algorithm}

Cascade control control is often used for experimental kite control, is [19, 23], designed in such a way as to produce a repetitive figure-of-eight pattern for the kite's position. This type of control was successfully tested in extensive sea trials $[19]^{2}$. To serve as a basis for comparison, we chose to implement the simple cascade approach proposed by [23] on the benchmark. A low-level controller regulates the heading-angle $\psi$. Although [23] uses a proportional low-level controller, we added an integral term for better performance. Note that [23] uses the velocity angle as the controlled variable, whereas (like [19]) we use the heading angle $\psi$. In any case, these angles are almost the same during crosswind flight. A reference value for $\psi$ is provided by the path-planning master controller. A block diagram of the control scheme is shown in Figure 7. The path-planning algorithm navigates the kite alternately between two points, by providing a reference heading angle that points at the current target. The kite will tend to fly a figure-of-eight trajectory between these two points, as illustrated in Figure 8. The gains of the low-level controller and the cut-off frequencies of the various filters (see [23] for more details) are fixed (through manual tuning using the simulator) at values that were found to give a good performance for tracking $\psi_{\text {ref }}$. In addition, we assume that the target points are aligned in real time with (i.e. symmetric with respect to) the wind experienced by the plant using the algorithm proposed by [39]. This leaves our implementation with two tuning parameters: $z_{\operatorname{targ}}$ is the height of the points, and $w_{\operatorname{targ}}$ is the distance (in a straight line) between them.

The average line tension $\bar{T}(200 \mathrm{~s})$ that can be achieved for the plant, with all possible combinations of the tuning parameters that ensure the kite does not crash into the ground, is shown in Figure 9. In the very best case, the average line tension $32.4 \mathrm{kN}$ can be achieved, about $18 \%$ less than the plant optimal value of $39.61 \mathrm{kN}$. The abrupt target-point changes cause the input signal pro-

\footnotetext{
${ }^{2}$ Recent publications contain promising preliminary results for more advanced pathfollowing controllers [31, 34, 11]
} 
duced by this controller to vary rapidly, which is undesirable. Furthermore, due to the heuristic nature of the algorithm, it is impossible to precisely control the kite trajectory, which varies significantly between orbits. During a very small percentage of orbits, the kite can violate the altitude constraint, even if it otherwise remains distant from it.

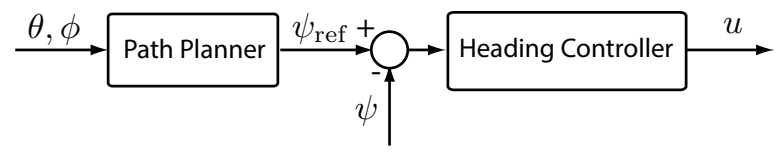

Figure 7: A simplified block diagram of the scheme proposed by [23].

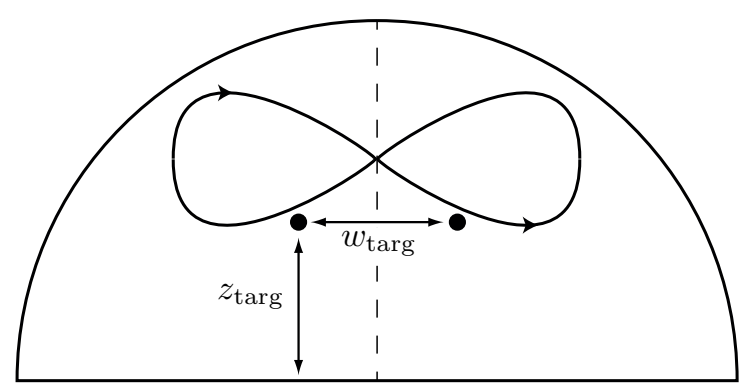

Figure 8: Illustration of the tuning parameters for our implementation of the point-to-point scheme proposed by [23]. The semi-circle is perpendicular to the wind, and shows the limits of the 'wind window', namely, the quarter-sphere on which the kite can fly.

\section{Conclusion}

This paper presents a relevant and challenging benchmark problem for advanced control and optimization techniques. The article is intended to be a stand-alone resource, and is aimed at the general, non kite specialist, control and optimization community. The control problem is currently of industrial relevance, as kite power is evolving into a promising wind-power technology. Experimental studies from the literature, industrial data, and the authors' experience in experimental kite control ensure that the benchmark problem reproduces a number of real-life challenges, namely fast nonlinear dynamics, stochastic disturbances, measurement noise and plant-model mismatch. The main contribution is to combine the ever-widening literature in this field into a coherent benchmark scenario. The actual modeling equations are taken from the existing literature, although they are presented in a slightly novel fashion. Nonetheless, it is worth noting that the experimentally validated wind turbulence model used in the simulated reality appears to have never been used in the field of kite power.

All the equations necessary to reproduce the simulated reality are given in this article and, in addition, a Simulink implementation of the plant is provided. Importantly, we were able to compute the optimal solution for the plant, which 


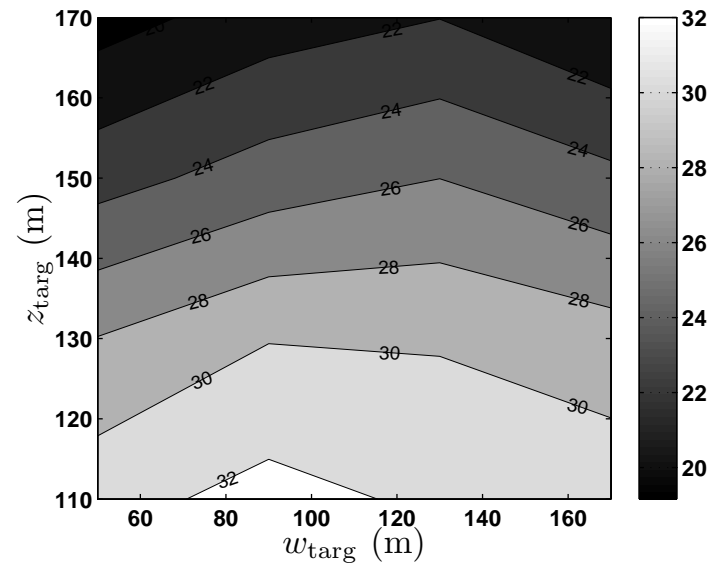

Figure 9: The average line tension $\bar{T}(200 \mathrm{~s})$ for the benchmark using the control scheme proposed by [23], for different values of $z_{\text {targ }}$ and $w_{\text {targ }}$.

facilitates the benchmarking process. A simple control strategy that is currently employed in practice was tested on the plant. On the benchmark simulation, this was found not only to be relatively inefficient, but also to provide only loose control over the kite path. The significant variability of the kite path obtained using this algorithm makes it difficult to guarantee that the height constraint is always respected. Of course, this absolutely does not imply that this simple algorithm will perform badly in reality, but in the simulation scenario it can certainly be improved upon. This opens the door for more advanced control techniques ${ }^{3}$ that, hopefully, can close the optimality gap, while ensuring smooth inputs and rigorous constraint satisfaction.

\footnotetext{
${ }^{3}$ The application of a two-layer optimizing control scheme to the simulation benchmark can be found in [13]. The simulation benchmark was an essential development stepping stone leading to the optimal control of an experimental system [14].
} 


\section{Nomenclature}

\begin{tabular}{|c|c|}
\hline$r$ & line length, m \\
\hline$A$ & area of kite, $\mathrm{m}^{2}$ \\
\hline$d$ & wingspan, m \\
\hline$m$ & mass of kite, $\mathrm{kg}$ \\
\hline$g$ & gravitational constant, $\mathrm{m} \cdot \mathrm{s}^{-2}$ \\
\hline$\rho$ & air density, $\mathrm{kg} \cdot \mathrm{m}^{-3}$ \\
\hline$T$ & line tension, $\mathrm{N}$ \\
\hline $\bar{T}$ & average line tension, $\mathrm{N}$ \\
\hline$x, y, z$ & cartesian co-ordinates for (inertial) $G$ frame, $\mathrm{m}$ \\
\hline$\theta, \phi, r$ & spherical co-ordinates for $G$ frame, rad, rad, m \\
\hline$\theta_{0}, \phi_{0}, \omega_{0}^{\theta}, \omega_{0}^{\phi}$ & plant initial position and velocity, $\mathrm{rad}, \mathrm{rad}, \mathrm{rad} \cdot \mathrm{s}^{-1}, \mathrm{rad} \cdot \mathrm{s}^{-1}$ \\
\hline $\mathbf{p}$ & kite position vector (in $G$ ), $\mathrm{m}$ \\
\hline$\psi$ & heading angle, rad \\
\hline$\tilde{\psi}$ & wrapped heading angle, rad \\
\hline$\psi_{\text {ref }}$ & set point for the heading angle, rad \\
\hline$t$ & time, $\mathrm{s}$ \\
\hline$u$ & set point for the steering deflection, $\mathrm{m}$ \\
\hline $\bar{u}$ & steering deflection, $\mathrm{m}$ \\
\hline $\bar{u}_{0}$ & plant initial steering deflection, $\mathrm{m}$ \\
\hline$u_{\max }$ & maximum value of $u, \mathrm{~m}$ \\
\hline$\tau_{\mathrm{u}}$ & steering actuator time constant, $\mathrm{s}$ \\
\hline$\mu$ & angle between the tether and the pitch axis, rad \\
\hline$\eta$ & rotation around tether to satisfy infinite-tail assumption, rad \\
\hline$\alpha$ & angle of attack, rad \\
\hline $\mathbf{F}$ & total force on kite, $\mathrm{N}$ \\
\hline $\mathbf{F}_{\text {aero }}$ & aerodynamic force on kite, $\mathrm{N}$ \\
\hline $\mathbf{F}_{\mathrm{g}}$ & gravitational force on kite, $\mathrm{N}$ \\
\hline$\hat{\mathbf{e}}_{\theta}$ & UV (unit vector) for an incremental change in $\theta$ \\
\hline$\hat{\mathbf{e}}_{\phi}$ & UV for an incremental change in $\phi$ \\
\hline$\hat{\mathbf{e}}_{\text {down }}$ & UV pointing toward the origin \\
\hline$\hat{\mathbf{e}}_{f}$ & UV opposing $\mathbf{w}_{\text {ap }}$ \\
\hline$\hat{\mathbf{e}}_{0}$ & UV perpendicular to the apparent wind \\
\hline$\hat{\mathbf{e}}_{\text {pitch }}$ & UV aligned with the kite right wing \\
\hline$\hat{\mathbf{e}}_{\text {roll }}$ & UV fixed to the kite, pointing forward \\
\hline $\mathbf{y}$ & vector of measured variables \\
\hline $\mathbf{y}_{\mathrm{N}}$ & vector of noisy measured variables \\
\hline$k$ & sampling counter \\
\hline$T_{\mathrm{s}}$ & sampling time, $\mathrm{s}$ \\
\hline
\end{tabular}




\begin{tabular}{|ll|}
$C_{\mathrm{L}}$ & lift coefficient, - \\
$C_{\mathrm{D}}$ & drag coefficient, - \\
$C_{\mathrm{L}, 0}, C_{\mathrm{L}, 1}, C_{\mathrm{D}, 0}, C_{\mathrm{D}, 2}$ & constants in the expressions for $C_{\mathrm{L}}$ and $C_{\mathrm{D}}$ \\
$\mathbf{w}$ & wind vector, $\mathrm{m} \cdot \mathrm{s}^{-1}$ \\
$w$ & wind speed $\mathrm{m} \cdot \mathrm{s}^{-1}$ \\
$\mathbf{w}_{\mathrm{a}}$ & apparent wind vector, $\mathrm{m} \cdot \mathrm{s}^{-1}$ \\
$\mathbf{w}_{\mathrm{ap}}$ & projection of $\mathbf{w}_{\mathrm{a}}$ onto the plane tangent to the sphere, $\mathrm{m} \cdot \mathrm{s}^{-1}$ \\
$w_{\mathrm{ap}}$ & magnitude of $\mathbf{w}_{\mathrm{ap}} \mathrm{m} \cdot \mathrm{s}^{-1}$ \\
$\chi$ & angle between the wind and the x-axis, degrees \\
$z_{\text {ref }}$ & reference altitude for wind law, $\mathrm{m}$ \\
$w_{\text {ref }}$ & RMS of the wind speed at reference altitude, $\mathrm{m} \cdot \mathrm{s}^{-1}$ \\
$a$ & exponent in the wind law \\
$w_{\mathrm{N}}$ & stochastic wind-speed component, $\mathrm{m} \cdot \mathrm{s}^{-1}$ \\
$\sigma_{\mathrm{w}}$ & standard deviation of $w_{\mathrm{N}}, \mathrm{m} \cdot \mathrm{s}^{-1}$ \\
$T_{\mathrm{w}}$ & update period of $w_{\mathrm{N}}, \mathrm{s}$ \\
$k_{\sigma, \mathrm{w}}$ & environmental parameter in the wind turbulence model, - \\
$L_{\mathrm{w}}$ & turbulence length, $\mathrm{m}$ \\
$H_{\mathrm{F}}, \tau_{\mathrm{F}}, K_{\mathrm{F}}$ & transfer function, time constant, gain of the turbulence shaping filter, - \\
$\theta_{\mathrm{N}}, \phi_{\mathrm{N}}, \psi_{\mathrm{N}}$ & additive measurement noises for $\theta, \phi, \psi, \mathrm{rad}$ \\
$\sigma_{\theta}, \sigma_{\phi}, \sigma_{\psi}$ & standard deviations of measurement noises on $\theta, \phi, \psi$, rad \\
$g_{\mathrm{s}}$ & steering constant for the model, rad $\cdot \mathrm{m}^{-2}$ \\
$c$ & steering penalty for the model, $\mathrm{m}^{-2}$ \\
$E_{0}$ & maximum lift-to-drag ratio for the model, - \\
$E$ & lift-to-drag ratio for the model, - \\
$w_{0}$ & fixed wind speed for the model, $\mathrm{m} \cdot \mathrm{s}^{-1}$ \\
$z_{\mathrm{min}}$ & minimum permissible altitude, $\mathrm{m}$ \\
$z_{\text {targ }}$ & target-point height, $\mathrm{m}$ \\
$w_{\text {targ }}$ & distance between the two target points, $\mathrm{m}$ \\
\hline &
\end{tabular}

\section{A Further Modeling Details for the Simulated Reality}

\section{A.1 Lift and drag coefficients}

The lift and drag coefficients vary considerably depending on the kite being considered. To facilitate reproducing the benchmark, we propose simple analytic profiles:

$$
\begin{aligned}
& C_{\mathrm{D}}(\alpha)=C_{\mathrm{D}, 0}+C_{\mathrm{D}, 2} \alpha^{2}, \\
& C_{\mathrm{L}}(\alpha)=C_{\mathrm{L}, 0}+C_{\mathrm{L}, 1} \alpha .
\end{aligned}
$$

Note that assuming the lift coefficient is linear in the angle of attack is a significant simplification. This neglects stalling, a decrease of lift that occurs at high angles of attack. However, this assumption is reasonable, as during dynamic kite flight with reasonably strong winds, stalling rarely occurs in practice. The resulting lift-to-drag ratio $C_{L} / C_{D}$, shown in Figure 10, is similar to the curve for a medium performance kite. 


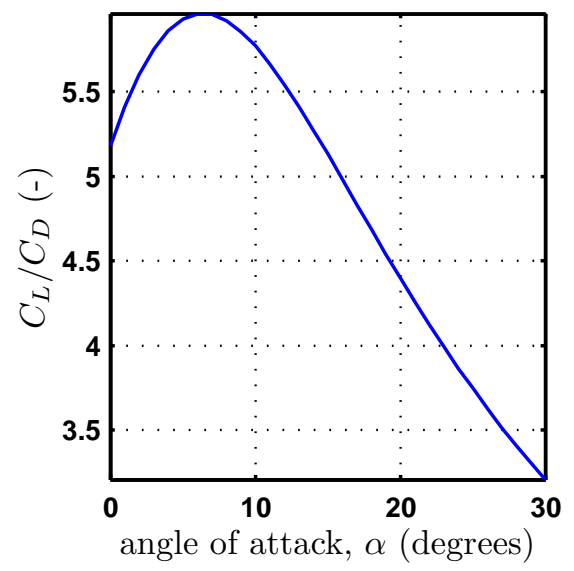

Figure 10: The lift-to-drag ratio for the plant.

\section{A.2 Wind model}

In the simulated reality, the wind vector $\mathbf{w}$ blows horizontally, at an angle $\chi$ to the $\mathrm{x}$-axis:

$$
\mathbf{w}=w\left(\hat{\mathbf{e}}_{\mathrm{x}} \cos \chi+\hat{\mathbf{e}}_{\mathrm{y}} \sin \chi\right) .
$$

The wind speed $w$ is an increasing function of the kite altitude. This so-called 'wind shear' is modeled using the classic power law [3]:

$$
w=\left(w_{\text {ref }}+w_{\mathrm{N}}\right)\left(z / z_{\text {ref }}\right)^{a}
$$

where $a$ is the surface friction coefficient, $w_{\text {ref }}$ is the reference wind speed at the reference altitude $z_{\text {ref }}$, and $w_{\mathrm{N}}$ is the stochastic wind turbulence. The windshear profile is shown in Figure 11.

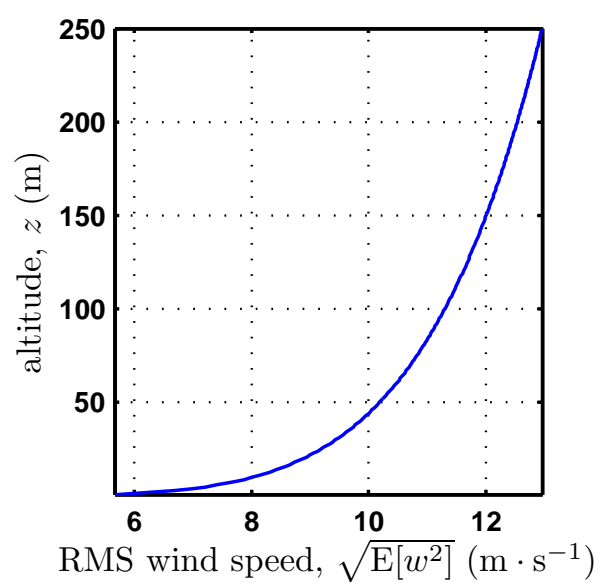

Figure 11: Wind profile for the plant. 
The wind turbulence, $w_{\mathrm{N}}$, is generated using a slightly simplified version of the established turbulence model proposed by [37], which is commonly used in wind-turbine simulations. This model, which was derived from a large quantity of experimental data, describes the short-term wind-speed variations (gusts) that occur on a scale of seconds and minutes due to turbulence in the wind flow. Complete wind models also take into account the long-term wind variations that occur on a scale of hours and days due to meteorological effects [33]. In the medium term, over a period of up to an hour, the mean wind speed can be considered constant [37]. As the focus of this benchmark is control and optimization over a short time period (several minutes), only a short-term turbulence model is used, which is depicted in Figure 12. The model is driven

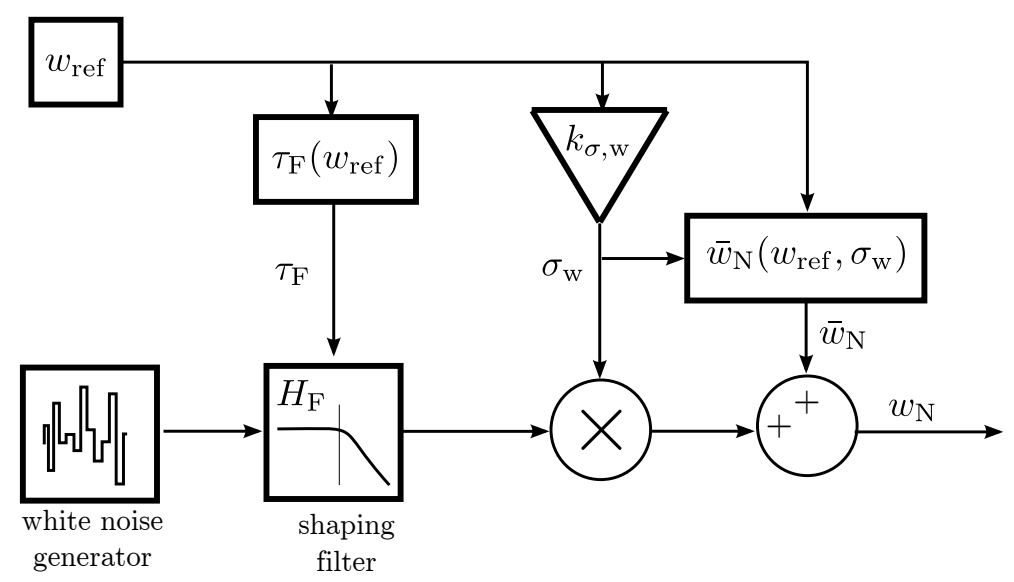

Figure 12: Wind turbulence generator for the plant.

by a Gaussian white-noise generator of unit variance and sampling period $T_{\mathrm{w}}$. The white-noise signal is passed through a first-order ${ }^{4}$ shaping filter with the following transfer function:

$$
H_{\mathrm{F}}(j \omega)=\frac{K_{\mathrm{F}}}{1+j \omega \tau_{\mathrm{F}}},
$$

where $K_{\mathrm{F}}$ is the filter gain, and $\tau_{\mathrm{F}}$ is the time constant. The time constant is given by

$$
\tau_{\mathrm{F}}=\frac{L_{\mathrm{w}}}{w_{\mathrm{ref}}}
$$

where the turbulence length $L_{\mathrm{w}}$ is an environmental parameter that depends on the surrounding terrain. The gain $K_{\mathrm{F}}$ is calculated in order to ensure the filter output has unity variance:

$$
K_{\mathrm{F}}=\sqrt{1.49 \frac{\tau_{\mathrm{F}}}{T_{\mathrm{w}}}}
$$

The filter output is scaled by the reference wind speed and the environmental parameter $k_{\sigma, \mathrm{w}}$. The standard deviation of the turbulence signal $w_{\mathrm{N}}$ is thus:

$$
\sigma_{\mathrm{w}}=k_{\sigma, \mathrm{w}} \cdot w_{\mathrm{ref}}
$$

\footnotetext{
${ }^{4}[37]$ actually used an irrational shaping filter; the first-order approximation used here yields almost identical results.
} 
Finally, the mean value of the turbulence component is calculated such that the RMS (root mean square) value of $w_{\text {ref }}+w_{\mathrm{N}}$ is $w_{\text {ref }}$ :

$$
\bar{w}_{\mathrm{N}}=-\frac{\sigma_{\mathrm{w}}}{2 w_{\mathrm{ref}}} .
$$

The parameter values used in the wind-shear model and the wind-turbulence model are given in Table 1 . They agree with the experimental values given by [3] and [37], and correspond to a medium strength wind blowing over a flat, grassy terrain. One realization of the wind turbulence is shown in Figure 13.

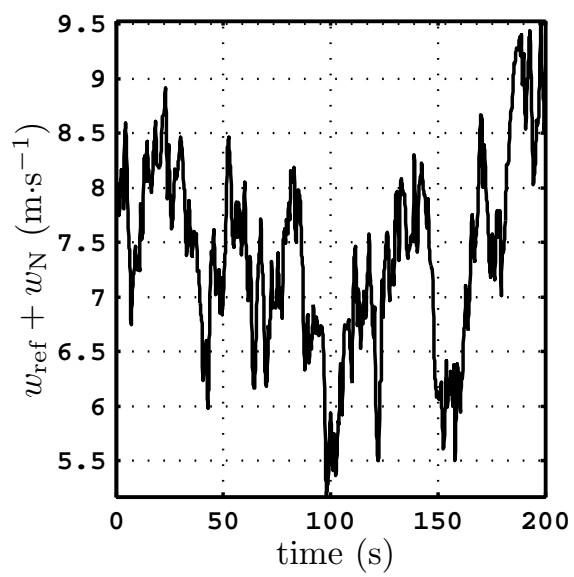

Figure 13: One realization of the stochastic wind speed for the simulated reality.

\section{References}

[1] U. Ahrens, M. Diehl, and R. Schmehl, editors. Airborne Wind Energy. Springer, Berlin, 2013.

[2] Ampyx Power. http://www. ampyxpower.com.

[3] C. L. Archer. An introduction to meteorology for airborne wind energy. In Ahrens et al. [1], pages 81-94.

[4] I. Argatov and R. Silvennoinen. Energy conversion efficiency of the pumping kite wind generator. Renewable Energy, 35(5):1052-1060, 2010.

[5] J. H. Baayen and W. J. Ockels. Tracking control with adaption of kites. Control Theory App., IET, 6(2):182-191, 2012.

[6] A. Bosch, R. Schmehl, P. Tiso, and D. Rixen. Nonlinear aeroelasticity, flight dynamics and control of a flexible membrane traction kite. In Ahrens et al. [1], pages 307-323.

[7] J. Breukels. An Engineering Methodology for Kite Design. PhD thesis, Delft Universtiy of Technology, 2010. 
[8] J. Breukels, R. Schmehl, and W. Ockels. Aeroelastic simulation of flexible membrane wings based on multibody system dynamics. In Ahrens et al. [1], pages 287-305.

[9] M. Canale, L. Fagiano, and M. Milanese. High altitude wind energy generation using controlled power kites. IEEE Trans. on Control Systems Tech., 18(2):279-293, 2010.

[10] B. Chachuat, B. Srinivasan, and D. Bonvin. Adaptation strategies for realtime optimization. Comp. Chem. Eng., 33(10):1557-1567, 2009.

[11] S. Costello. Real-Time Optimization via Directional Modifier Adaptation, with Application to Kite Control. PhD thesis, \# 6571, EPFL, Lausanne, 2015 .

[12] S. Costello, G. François, and D. Bonvin. Real-time optimization for kites. In Proc. of the $5^{\text {th }}$ IFAC Workshop on Periodic Control Systems (PSYCO), pages 64-69, 2013.

[13] S. Costello, G. François, and D. Bonvin. Directional real-time optimization applied to a kite-control simulation benchmark. In European Control Conference, pages 1594-1601, 2015.

[14] S. Costello, G. François, and D. Bonvin. Real-time optimizing control of an experimental crosswind power kite. Submitted to IEEE Trans. on Control Systems Tech., 2016.

[15] G. M. Dadd, D. A. Hudson, and R. A. Shenoi. Comparison of two kite force models with experiment. J. of Aircraft, 47:212-224, 2010.

[16] G. M. Dadd, D. A. Hudson, and R. A. Shenoi. Determination of kite forces using three-dimensional flight trajectories for ship propulsion. Renewable Energy, 36(10):2667-2678, 2011.

[17] M. Diehl. Real Time Optimization for Large Scale Nonlinear Processes. PhD thesis, Ruprecht-Karls-Universität Heidelberg, 2001.

[18] M. Erhard, G. Horn, and M. Diehl. A quaternion-based model for optimal control of the skysails airborne wind energy system. arXiv preprint arXiv:1508.05494, 2015.

[19] M. Erhard and H. Strauch. Control of towing kites for seagoing vessels. IEEE Trans. on Control Systems Tech., 21(5):1629-1640, 2013.

[20] M. Erhard and H. Strauch. Theory and experimental validation of a simple comprehensible model of tethered kite dynamics used for controller design. In Ahrens et al. [1], pages 141-165.

[21] M. Erhard and H. Strauch. Flight control of tethered kites in autonomous pumping cycles for airborne wind energy. Control Eng. Practice, 40(0):1326,2015 .

[22] L. Fagiano and M. Milanese. Airborne wind energy: An overview. In Proc. American Control Conference, pages 3132-3143, 2012. 
[23] L. Fagiano, A. U. Zgraggen, M. Morari, and M. Khammash. Automatic crosswind flight of tethered wings for airborne wind energy: Modeling, control design, and experimental results. IEEE Trans. on Control Systems Tech., 22(4):1433-1447, 2014.

[24] F. Gohl and R. H. Luchsinger. Simulation based wing design for kite power. In Ahrens et al. [1], pages 325-338.

[25] S. Gros and M. Diehl. Modeling of airborne wind energy systems in natural coordinates. In Ahrens et al. [1], pages 181-203.

[26] G. Horn, S. Gros, and M. Diehl. Numerical trajectory optimization for airborne wind energy systems described by high fidelity aircraft models. In Ahrens et al. [1], pages 205-218.

[27] B. Houska. Robustness and stability optimization of open-loop controlled power generating kites. Master's thesis, Ruprecht-Karls-Universität Heidelberg, 2007.

[28] B. Houska and M. Diehl. Optimal control of towing kites. In Proc. 45th IEEE Conf. on Decision and Control, pages 2693-2697, 2006.

[29] B. Houska and M. Diehl. Optimal control for power generating kites. In Proc. 9th European Control Conference, pages 3560-3567, 2007.

[30] A. Ilzhöfer, B. Houska, and M. Diehl. Nonlinear MPC of kites under varying wind conditions for a new class of large-scale wind power generators. Int. J. Robust and Nonlinear Control, 17(17):1590-1599, 2007.

[31] C. Jehle and R. Schmehl. Applied tracking control for kite power systems. J. Guidance, Control, and Dynamics, 37(4):1211-1222, 2014.

[32] M. L. Loyd. Crosswind kite power. J. Energy, 4(3):106-111, May 1980.

[33] C. Nichita, D. Luca, B. Dakyo, and E. Ceanga. Large band simulation of the wind speed for real time wind turbine simulators. IEEE Trans. on Energy Conversion, 17(4):523-529, 2002.

[34] R. Ruiterkamp and S. Sieberling. Description and preliminary test results of a six degrees of freedom rigid wing pumping system. In Ahrens et al. [1], pages 443-458.

[35] SKP. http://www.swisskitepower.ch.

[36] M. Vukov, S. Gros, G. Horn, G. Frison, K. Geebelen, J. B. Jørgensen, J. Swevers, and M. Diehl. Real-time nonlinear MPC and MHE for a largescale mechatronic application. Control Eng. Practice, 45:64-78, 2015.

[37] E. Welfonder, R. Neifer, and M. Spanner. Development and experimental identification of dynamic models for wind turbines. Chem. Eng. Process., $5(1): 63-73,1997$.

[38] P. Williams, B. Lansdorp, and W. Ockesl. Optimal crosswind towing and power generation with tethered kites. J. Guidance, Control, and Dynamics, 31(1):81-93, 2008. 
[39] A. U. Zgraggen, L. Fagiano, and M. Morari. On real-time optimization of airborne wind energy generators. In Proc. 52 ${ }^{\text {nd }}$ IEEE Conf. on Decision and Control, pages 385-390, Florence, 2013.

[40] A. U. Zgraggen, L. Fagiano, and M. Morari. Real-time optimization and adaptation of the crosswind flight of tethered wings for airborne wind energy. IEEE Trans. on Control Systems Tech., 23(2):434-448, 2015.

[41] A. U. Zgraggen, L. Fagiano, and M. Morari. Automatic retraction and full-cycle operation for a class of airborne wind energy generators. IEEE Trans. on Control Systems Tech., 24(2):594-608, 2016. 